\title{
DECOMPOSITION OF FUNCTION-LATTICES
}

\author{
S. D. SHORE
}

Abstract. We give a simple direct proof of the theorem (due to Kaplansky-Blair-Burrill) that the lattice $C(X, K)$ of all continuous functions defined on the topological space $X$ with values in the chain $K$ can be decomposed iff $X$ contains an open-and-closed subset.

For any topological space $X$, let $C(X, K)$ denote the lattice of all $K$-valued continuous functions defined on $X$, where $K$ is any nonsingleton totally ordered set with the order topology. Clearly, if $A$ is any open-and-closed subspace of $X$, then $C(X, K)$ is lattice isomorphic to the direct product $C(A, K) \times C(X \backslash A, K)$. Improving a technique of Kaplansky [2], Blair and Burrill [1] have shown that a converse holds. We give a simple alternative proof of this result which, in contrast to the proofs of Kaplansky and Blair-Burrill, avoids use of the axiom of choice. For this observation and several other suggestions for improving the presentation we are grateful to the referee.

A sublattice $L \subseteq C(X, K)$ is adequate provided that, for each $x \in X$, there are functions $f, g \in L$ such that $f(x) \neq g(x)$.

THEOREM. If an adequate sublattice $L$ of $C(X, K)$ is lattice isomorphic to the direct product $L_{1} \times L_{2}$ of lattices $L_{1}$ and $L_{2}$, then there is an openand-closed subset $A \subseteq X$ such that $L_{1}$ is lattice isomorphic to $\{f \mid A: f \in L\}$ and $L_{2}$ is lattice isomorphic to $\{f \mid(X \backslash A): f \in L\}$.

We first establish a

Lemma. Let $L_{1}$ and $L_{2}$ be lattices and $K$ be a totally ordered set. If $\alpha: L_{1} \times L_{2} \rightarrow K$ is a lattice homomorphism, then one of the following holds:

(1) For any $k, k^{\prime} \in L_{2}, \alpha(l, k)=\alpha\left(l, k^{\prime}\right)$ for any $l \in L_{1}$.

(2) For any $l, l^{\prime} \in L_{1}, \alpha(l, k)=\alpha\left(l^{\prime}, k\right)$ for any $k \in L_{2}$.

Moreover, if $\alpha$ is not constant, then precisely one of these holds.

Proof. Note that (1) is equivalent to:

$\left(1^{\prime}\right)$ For any $k, k^{\prime} \in L_{2}, \alpha\left(l_{0}, k\right)=\alpha\left(l_{0}, k^{\prime}\right)$ for some $l_{0} \in L_{\mathbf{1}}$.

This follows from the observation that

Received by the editors September 22, 1969.

AMS 1968 subject classifications. Primary 4625; Secondary 0630.

Key words and phrases. Lattices of chain-valued functions, adequate sublattices, homomorphism, connectedness. 


$$
\begin{aligned}
\alpha(l, k) & =\alpha\left(\left(\left(l_{0}, k\right) \wedge\left(l, k \vee k^{\prime}\right)\right) \vee\left(l, k \wedge k^{\prime}\right)\right) \\
& =\alpha\left(\left(\left(l_{0}, k^{\prime}\right) \wedge\left(l, k \vee k^{\prime}\right)\right) \bigvee\left(l, k \wedge k^{\prime}\right)\right)=\alpha\left(l, k^{\prime}\right) .
\end{aligned}
$$

Similarly, (2) is equivalent to:

(2') For any $l, l^{\prime} \in L_{1}, \alpha\left(l, k_{0}\right)=\alpha\left(l^{\prime}, k_{0}\right)$ for some $k_{0} \in L_{2}$.

Now, assume that condition (2) fails. Then, there exist $l, l^{\prime} \in L_{1}$ such that $\alpha(l, k) \neq \alpha\left(l^{\prime}, k\right)$ for any $k \in L_{2}$. To show that condition (1) holds, consider any $k, k^{\prime} \in L_{2}$. Then,

$$
\alpha\left(l \wedge l^{\prime}, k \vee k^{\prime}\right) \vee \alpha\left(l \vee l^{\prime}, k \wedge k^{\prime}\right)=\alpha\left(l \vee l^{\prime}, k \vee k^{\prime}\right)
$$

Since $K$ is totally ordered and $\alpha\left(l \wedge l^{\prime}, k \bigvee k^{\prime}\right)=\alpha\left(l \bigvee l^{\prime}, k \bigvee k^{\prime}\right)$ implies that $\alpha\left(l, k \bigvee k^{\prime}\right)=\alpha\left(l^{\prime}, k \bigvee k^{\prime}\right)$ (a contradiction), we conclude that $\alpha\left(l \bigvee l^{\prime}, k \wedge k^{\prime}\right)=\alpha\left(l \bigvee l^{\prime}, k \bigvee k^{\prime}\right)$. Hence, $\alpha\left(l \bigvee l^{\prime}, k\right)=\alpha\left(l \bigvee l^{\prime}, k^{\prime}\right)$ so that conditions $\left(1^{\prime}\right)$ and (1) hold. Evidently, both conditions hold iff $\alpha$ is constant.

Proof of the Theorem. Let $L$ be an adequate sublattice of $C(X, K)$ and $\psi: L_{1} \times L_{2} \rightarrow L$ be a lattice isomorphism. For each $x \in X$, the lattice homomorphism $\varphi_{x}: L \rightarrow K$, defined by $\varphi_{x}(f)=f(x)$, is not constant. From the preceding lemma $\varphi_{x} \circ \psi: L_{1} \times L_{2} \rightarrow K$ satisfies one, and only one, of the conditions (1) and (2). Define $A=\left\{x \in X: \varphi_{x} \circ \psi\right.$ satisfies condition (1) $\}$. It follows easily that $A$ and $X \backslash A$ are disjoint closed sets. Finally, define $\theta: L_{1} \rightarrow\{f \mid A: f \in L\}$ by $\theta(l)=f \mid A$, where $f=\psi\left(l, k_{0}\right)$ for some $k_{0} \in L_{2}$. It follows directly that $\theta$ is a lattice isomorphism. Similarly, one considers $X \backslash A$ so that the proof of the theorem is complete.

Remarks. An easy corollary is that a topological space $X$ is connected iff, for any totally ordered set $K$, there is no adequate sublattice $L \subseteq C(X, K)$ which is lattice isomorphic to the direct product $L_{1} \times L_{2}$ of two lattices $L_{1}$ and $L_{2}$, neither of which is a singleton. Hence, a topological space $X$ is connected iff every extension of $X$ is connected (where an extension of $X$ is any topological space that contains $X$ as a dense subspace).

\section{REFERENCES}

1. R. L. Blair and C. W. Burrill, Direct decompositions of lattices of continuous functions, Proc. Amer. Math. Soc. 13 (1962), 631-634. MR 25 \#2440.

2. I. Kaplansky, Lattices of continuous functions, Bull. Amer. Math. Soc. 53 (1947), 617-623. MR 8, 587.

University of New Hampshire, Durham, New Hampshire 03824 